\title{
Resistive hose growth of intense ion beams propagating in air
}

\author{
D. V. Rose, ${ }^{*}$ T. C. Genoni, and D. R. Welch \\ Voss Scientific, LLC, Albuquerque, New Mexico 87108, USA \\ M. G. Mazarakis \\ Sandia National Laboratories, Albuquerque, New Mexico 87185, USA \\ (Received 17 February 2006; published 27 April 2006)
}

\begin{abstract}
The growth of the resistive hose instability for intense proton beams is examined using threedimensional particle-in-cell simulations. The simulation results are compared with a time-dependent model of resistive hose growth that uses a spread-mass formulation and a time-dependent conductivity model. Radius tailoring of the beam head is shown to suppress high-frequency instability growth. In addition, the effects of a reduced-density plasma channel on the growth of the resistive hose instability is calculated.
\end{abstract}

DOI: 10.1103/PhysRevSTAB.9.044403

PACS numbers: $52.40 . \mathrm{Mj}, 52.65 . \mathrm{Rr}$

\section{INTRODUCTION}

Intense charged particle beams propagating in a background gas are known to be susceptible to the resistive hose instability. This instability has been extensively studied over the past 30 years [1-13], and transport of the beam in reduced-density channels has been studied as an aid to extending transport distances [14-20].

For a beam propagating in a neutral or weakly ionized gas with electric fields below $\sim 75 \mathrm{kV} / \mathrm{cm}$-atm, the plasma channel conductivity is set up by beam-impact ionization of the gas. The equilibrium conductivity increases with distance back into the body of the beam until recombination and/or plasma cooling (radiation, conduction, etc.) balances beam-impact ionization and/or heating. The beam pulse length is limited by the head-to-tail growth of the resistive hose instability. The maximum asymptotic growth rate of this instability is [1] $\max [\operatorname{Im}(\omega)]=\omega_{i \max } \simeq$ 0.7 . The trajectory of the fastest growing wave packet is $Z / T \simeq 6.33[1]$ or

$$
\tau \simeq k_{m B} \tau_{B} z / 6.33
$$

Dimensionless time and axial distances are defined as $T=$ $\tau / \tau_{B}$ and $Z=k_{m B} z$, where $\tau$ is the characteristic beam time, $\tau_{B}=\pi \sigma_{0} a_{0}^{2} / 2 c^{2}$ is the dipole decay time, $\sigma_{0}$ is the plasma channel conductivity, $k_{m B}=\sqrt{2 e \beta I_{b} / \gamma m_{B} c v_{z}^{2} a_{0}^{2}}$ is the beam betatron wave number, $a_{0}$ is the beam radius, $I_{b}$ is the beam current and $c$ is the speed of light.

The equilibrium plasma conductivity generated by the interaction of the ion beam and return currents in the gas can be related to the beam current through [21]

$$
\frac{4 \pi \sigma_{0}}{c}\left(\mathrm{~cm}^{-1}\right) \simeq 167 \sqrt{\frac{I_{b}(\mathrm{kA})}{10 a_{0}^{2}}} .
$$

This relation is based on the balance between impact

*Electronic address: David.Rose@ vosssci.com ionization and dissociative recombination assuming a 1-eV plasma electron temperature. Using Eq. (2) to eliminate $\sigma_{0}$ in the dipole decay time gives

$$
\tau_{B} \simeq 0.696 \times 10^{-9} \sqrt{\frac{I_{b}(\mathrm{kA})}{10}} a_{0} .
$$

The betatron wave number can then be rewritten as $k_{m B} \simeq$ $8.62 \times 10^{-3} \sqrt{I_{b}} / a_{0}$. Substituting these expressions for $\tau_{B}$ and $k_{m B}$ into Eq. (1) gives

$$
\tau(\mathrm{ns}) \simeq 30 I_{b}(\mathrm{kA}) z(\mathrm{~km}),
$$

and the peak growth factor occurs at $z(\mathrm{~km}) \simeq$ $\tau(\mathrm{ns}) / 30 I_{b}(\mathrm{kA})$. Thus, for a $10-\mathrm{kA}$ beam at a distance $0.1 \mathrm{~km}$, the largest offset occurs at $\tau=30 \mathrm{~ns}$ and for a 40-kA beam, $\tau=120 \mathrm{~ns}$.

Three-dimensional particle-in-cell simulations are carried out to examine the growth and saturation of the resistive hose instability for intense proton beams. The simulations are compared with a semianalytic model of the time-dependent hose growth in a background gas. A scalar conductivity model [21] is used which is valid for high gas pressures where significant net currents are expected (due to recombination). We expect the analytic model presented in Sec. II to be independent of the processes that lead to net current generation, making this work applicable to a variety of other beam transport problems. These applications include transport of intense light-ion beams for inertial confinement fusion [22,23], a proposed scheme for long-distance propagation $(\gtrsim 100 \mathrm{~m})$ of heavy-ion beams from an accelerator to a reactor chamber as well as transport inside of the reactor chamber for heavy-ion inertial fusion energy [24]. The modeling techniques presented here have been applied to the study of high-current heavy-ion beam propagation over 5-m distances in laser initiated discharge channels [25].

A detailed semianalytic model for hose growth is presented in Sec. II along with some sample results. The 
particle-in-cell (PIC) simulation model used in this work is described in Sec. III. Three-dimensional PIC simulations of resistive hose growth are compared with the semianalytic model in Sec. IV. A summary of this work is given in Sec. V.

\section{ANALYTIC MODEL OF THE RESISTIVE HOSE INSTABILITY}

In this section we discuss analytic modeling of the resistive hose instability for intense ion beams. The goal is to develop a relatively simple and fast-running tool capable of correctly identifying trends in hose growth behavior with variation in beam parameters (energy, radius, and radius tailoring). The well-known spread-mass model is used and extended to include a variable channel conductivity profile and radius tailoring (detuning) from the head of the beam back to the body. The effects of plasma return current and channel perturbations were deemed beyond the scope of the present effort and were not included. In Sec. II A below, the basic spread-mass model equations are reviewed. In Sec. II B, the standard dispersion analysis is reviewed and then generalized to the case of an arbitrary (other than Bennett) conductivity profile. Finally, in Sec. IIC, we present results from numerical integration of the model equations generalized to account for the time evolution of the channel conductivity and for radius tailoring. Comparison of the model with results from the PIC simulations is presented in Sec. IV.

\section{A. Model equations}

We begin with the equations of Lee (Ref. [1]) for the spread-mass model of the resistive hose instability of a self-pinched relativistic beam:

$$
\begin{aligned}
& \frac{\partial^{2} Y_{\eta}}{\partial z^{2}}= 3 \eta\left(\frac{e \beta \pi}{\gamma M v_{z}^{2} I_{b}}\right) \\
& \times \int_{0}^{\infty} r d r\left[-Y_{\eta} \frac{d J_{0}}{d r} \frac{d A_{0}}{d r}+\frac{J_{0}}{r} \frac{\partial}{\partial r}\left(r A_{1}\right)\right], \\
& \frac{\partial}{\partial r}\left[\frac{1}{r} \frac{\partial}{\partial r}\left(r A_{1}\right)\right]-\frac{4 \pi \sigma(r, \tau)}{c^{2}} \frac{\partial A_{1}}{\partial \tau}=\frac{4 \pi}{c}\left\langle Y_{\eta}\right\rangle \frac{d J_{0}}{d r} .
\end{aligned}
$$

Equation (5) [see Eq. (95) of Ref. [1]] is the equation of motion of the centroid of beam disk $\eta(0 \leq \eta \leq 1) . J_{0}$ and $A_{0}$ are the equilibrium beam current and magnetic vector potential, respectively, and

$$
\begin{aligned}
& J_{0}(r)=\frac{I_{b}}{\pi a^{2}}\left(1+\frac{r^{2}}{a^{2}}\right)^{-2}, \\
& A_{0}(r)=-\frac{I_{b}}{c} \log \left(1+\frac{r^{2}}{a^{2}}\right),
\end{aligned}
$$

for a beam with a Bennett profile, radius $a$, and beam current $I_{b}$. The beam particle charge is $e$, mass $M$, and $v_{z} \simeq$ $\beta c$ in the paraxial-ray approximation. Equation (6) [see Eq. (100) of Ref. [1]] is the perturbed magnetic field $\left(A_{1}\right)$ equation with the channel conductivity $\sigma(r, \tau)$ written in general as an arbitrary function of radius and beam position (time) $\tau$ measured back from the head of the beam. We denote the mean of a quantity by the angle brackets; i.e.,

$$
\left\langle Y_{\eta}\right\rangle \equiv \int_{0}^{1} d \eta g(\eta) Y_{\eta}
$$

where $g(\eta)=6 \eta(1-\eta)$ is the weighting function for a Bennett beam in the spread-mass model. We define the betatron wave number $k_{\beta}$ and the magnetic decay time according to

$$
k_{\beta}^{2}=\frac{2 e \beta I_{b}}{\gamma M c v_{z}^{2} a_{0}^{2}}
$$

and

$$
\tau_{B}=\frac{\pi \sigma_{0} a_{0}^{2}}{2 c^{2}},
$$

respectively. In Eqs. (10) and (11), $a_{0}$ refers to the radius of the beam in the beam body (we will later consider the case of 3-to-1 radius tailoring from the beam head back to a constant value $a_{0}$.) We write the channel conductivity as follows:

$$
\sigma(r, \tau) \equiv \sigma_{0}(\tau) \bar{\sigma}(r, \tau)
$$

where $\sigma_{0}(\tau)$ is the on-axis conductivity and $\bar{\sigma}(r, \tau)$ describes the evolution of the conductivity profile. In Eq. (11), $\sigma_{0} \equiv \sigma_{0}(\tau \rightarrow \infty)$ is the on-axis conductivity in the body of the beam. Defining the following normalized variables,

$$
Z \equiv k_{\beta} z, \quad T \equiv \tau / \tau_{B}, \quad u \equiv r / a_{0},
$$

and performing the indicated substitutions and integrations (making use of integration by parts) in Eq. (5), our model equations for the beam disk centroid and perturbed magnetic field take the form

$$
\frac{\partial^{2} Y_{\eta}}{\partial Z^{2}}=-\eta \alpha^{2}(T)\left\{Y_{\eta}-\int_{0}^{\infty} \frac{6 u^{2} \alpha^{4}(T)}{\left[u^{2}+\alpha^{2}(T)\right]^{3}} \bar{A}_{1}(u) d u\right\}
$$

$$
\frac{\partial}{\partial u}\left[\frac{1}{u} \frac{\partial}{\partial u}\left(u \bar{A}_{1}\right)\right]-8 \bar{\sigma}(u, T) \frac{\partial \bar{A}_{1}}{\partial T}=\frac{-16 u \alpha^{2}(T)}{\left[u^{2}+\alpha^{2}(T)\right]^{3}}\left\langle Y_{\eta}\right\rangle,
$$

where $\bar{A}_{1}=c A_{1} / I_{b}$ is the normalized vector potential and $\alpha(T)=a(T) / a_{0}$ is the (in general time-varying) normalized beam radius.

\section{B. Dispersion analysis}

We consider perturbations of the form

$$
e^{i(\omega \tau+k z)}=e^{i(\Omega T+K Z)},
$$


where $\Omega=\omega \tau_{B}$ and $K=k / k_{\beta}$ are the normalized frequency and Doppler-shifted wave number, respectively.

\section{Bennett conductivity profile}

Lee has shown that for a constant beam radius, $\alpha(T)=$ 1 , and Bennett conductivity profile

$$
\bar{\sigma}(u, T)=\left(u^{2}+1\right)^{-2} .
$$

$A_{1}$ may be written in the form

$$
A_{1}=-D(Z, T) \frac{d A_{0}}{d u},
$$

and Eqs. (13) and (14) collapse to the simplified form

$$
\begin{gathered}
\frac{\partial Y_{\eta}}{\partial Z^{2}}=-\eta\left(Y_{\eta}-D\right), \\
\frac{\partial D}{\partial T}=\left\langle Y_{\eta}\right\rangle-D .
\end{gathered}
$$

Using the form in Eq. (15) for the perturbed quantities $\left(Y_{\eta}\right.$ and $D$ ) in Eqs. (17) and (18) yields the dispersion equation first derived by Lee [1]:

$$
i \Omega=\int_{0}^{1} 6 \eta(1-\eta) \frac{K^{2}}{\eta-K^{2}} d \eta .
$$

For a given beam slice $T$, instability growth is bounded according to

$$
\left\langle Y_{\eta}\right\rangle \leq C_{0} e^{\Omega_{0} T}=C_{0} e^{\Gamma},
$$

where $C_{0}$ is a constant and $\Omega_{0}$ is the maximum of the imaginary part of $\Omega$ for all real $K$ in Eq. (19). Lee finds
$\Omega_{0}=0.6902$ for $K_{r}=0.5218$. This peak growth occurs at a position downstream given approximately by

$$
\frac{Z}{T}=-\left(\frac{\partial \Omega_{i}}{\partial K}\right)_{\Omega_{i}=\Omega_{0}}=6.337
$$

As an example, consider a $10-\mathrm{kA}, 300-\mathrm{MeV}, 2-\mathrm{cm}$ radius proton beam propagating in air. Simulations using the channel conductivity model (Refs. $[21,26]$ ) and the above beam parameters predict

$$
\frac{4 \pi \sigma_{0}}{c} \sim 100 \mathrm{~cm}^{-1}
$$

Using Eq. (11) we get $\tau_{B} \sim 8.33 \times 10^{-10}$ s. For the beam slice at $\tau=50 \mathrm{~ns}, T=36$, and Eq. (19) gives a growth factor $\Gamma=24.8$, or approximately $25 e$-foldings.

\section{Arbitrary conductivity profile}

For conductivity profiles other than the Bennett, the simplification of Eq. (16) no longer pertains. We again set $\alpha(T)=1$ and consider an arbitrary (but constant in $T)$ radial conductivity profile $\bar{\sigma}(u)$. Under these conditions, Eqs. (13) and (14) may be combined to give

$$
\begin{aligned}
\frac{\partial}{\partial u}\left(\frac{1}{u} \frac{\partial F}{\partial u}\right)-8 i \Omega \bar{\sigma}(u) \frac{F}{u}= & \frac{-24 u}{\left(u^{2}+1\right)^{3}}\left\langle\frac{\eta}{\eta-K^{2}}\right\rangle \\
& \times \int_{0}^{\infty} \frac{4 u}{\left(u^{2}+1\right)^{3}} F(u) d u
\end{aligned}
$$

where $F(u) \equiv u \bar{A}_{1}(u)$. We discretize Eq. (22) setting $u=$ $n \Delta u, n=1,2,3, \ldots$ to obtain

$$
\begin{array}{r}
\frac{F(n+1)-2 F(n)+F(n-1)}{(n \Delta u)(\Delta u)^{2}}-\frac{F(n+1)-F(n-1)}{(n \Delta u)^{2} 2 \Delta u}-\frac{8 i \Omega F(n) \bar{\sigma}(n \Delta u)}{(n \Delta u)}+\frac{24(n \Delta u)}{\left[(n \Delta u)^{2}+1\right]^{3}}\left\langle\frac{\eta}{\eta-K^{2}}\right\rangle \\
\times\left\{\sum_{m} \frac{4(m \Delta u)}{\left[(m \Delta u)^{2}+1\right]^{3}} F(m) w t(m) \Delta u\right\}=0
\end{array}
$$

where $F(n) \equiv F(n \Delta u)$ and $w t(m)$ denotes weighting factors for approximate numerical integration (a Simpson's rule was used in the numerical calculations which follow).

Defining the vector $\bar{F}$ with components $F_{n} \equiv F(n)$, we obtain the infinite linear system

$$
\overline{\bar{A}} \bar{F}=0
$$

with the components of matrix $\overline{\bar{A}}$ given by

$$
\begin{aligned}
A_{n m}= & \frac{1}{(n \Delta u)(\Delta u)^{2}}\left(\delta_{n+1, m}-2 \delta_{n, m}+\delta_{n-1, m}\right)-\frac{1}{(n \Delta u)^{2} 2 \Delta u}\left(\delta_{n+1, m}-\delta_{n-1, m}\right)-\frac{8 i \Omega \bar{\sigma}(n \Delta u)}{(n \Delta u)} \delta_{n, m} \\
& +\frac{24(n \Delta u)}{\left[(n \Delta u)^{2}+1\right]^{3}}\left\langle\frac{\eta}{\eta-K^{2}}\right\rangle \frac{4 m \Delta u}{\left[(m \Delta u)^{2}+1\right]^{3}} w t(m) \Delta u .
\end{aligned}
$$


Truncation of the system at some large $n \Delta u$ (typically 20 or 30) gives a finite matrix $\overline{\bar{A}}$ and the dispersion equation becomes

$$
\operatorname{det} \overline{\bar{A}}=0 \text {. }
$$

As a benchmark, Eq. (26) was solved using a Bennett conductivity profile to obtain $\Omega_{0}=0.693$ and distance to peak growth $Z / T=6.34$, in close agreement with Lee's results from Eq. (19).

We note that the conductivity model previously mentioned predicts a profile more like a Lorentzian (square root of the Bennett) in the body of the beam. (We will return to this discussion in the next section.) We consider this profile:

$$
\bar{\sigma}(u)=\left(u^{2}+1\right)^{-1}
$$

and solve the dispersion equation to obtain $\Omega_{0}=0.247$ and $Z / T=2.67$. For this profile, Eq. (19) gives a peak growth factor $\Gamma=8.89$ for our beam slice at $\tau=50 \mathrm{~ns}-$ approximately $9 e$-foldings, a much more tolerable value than the 25 obtained with the Bennett assumption.

\section{Numerical integration of model equations}

In this section we report on results obtained by direct numerical integration of Eqs. (13) and (14) for several cases of interest. We test first the upper bounds on growth obtained from the dispersion analysis in the previous section for the constant Bennett and Lorentzian channel conductivity profiles. (In these calculations, each beam slice was divided into a large number of "spread-mass" disks, typically 40 , and a maximum radius of 50 beam radii was used. The response to a constant offset perturbation at $z=$ 0 was observed.) Results are shown in Fig. 1 for the Bennett conductivity profile. The observed growth factors [ $\ln$ (peak growth)] are plotted as a function of beam position and are consistent with the upper bounds calculated from Eq. (20). For $\tau=50 \mathrm{ns,} \mathrm{the} \mathrm{observed} \mathrm{peak} \mathrm{growth}$ factor was approximately 22.3 (as compared to an upper bound of nearly 25). The dispersion analysis also predicts a distance to peak growth (or, equivalently, the convection rate of the peak of the wave packet) of $Z / T=6.34$. The results shown in Fig. 2 from the numerical calculation of the model equations are again consistent with the dispersion analysis.

Results for the Lorentzian channel conductivity profile as shown in Figs. 3 and 4 and are again consistent with the dispersion analysis predictions. We see that for the Lorentzian channel, the peak growth factors and wave packet convection rates are considerably reduced from the Bennett case.

We turn now to a somewhat more realistic model of the beam and channel conductivity similar to that used in the simulations reported on in Sec. IV. We consider 3-to-1 radius tailoring from the head of the beam back to the body with a characteristic time of 4 ns, i.e.,

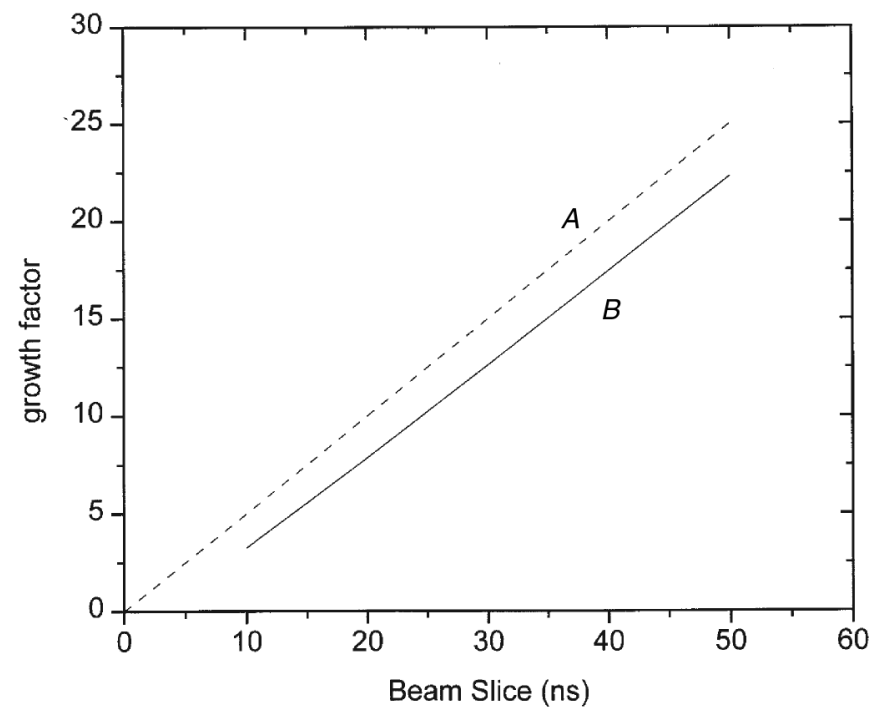

FIG. 1. For a Bennett conductivity profile, comparison of (A) upper bound for growth factor from dispersion analysis and $(B)$ observed growth factor from numerical integration of model equations.

$$
\alpha(\tau)=\frac{a(\tau)}{a_{0}}=1+2 e^{-\tau / \tau_{0}} \quad\left(\tau_{0}=4 \mathrm{~ns}\right) .
$$

The time evolution of the channel conductivity profile is determined by the differential equation

$$
\frac{d n_{e}}{d \tau}=C_{1} n_{b}-C_{2} n_{e}^{2}
$$

where $n_{e}$ is the channel electron density, $n_{b}$ is the beam density, and the channel conductivity is

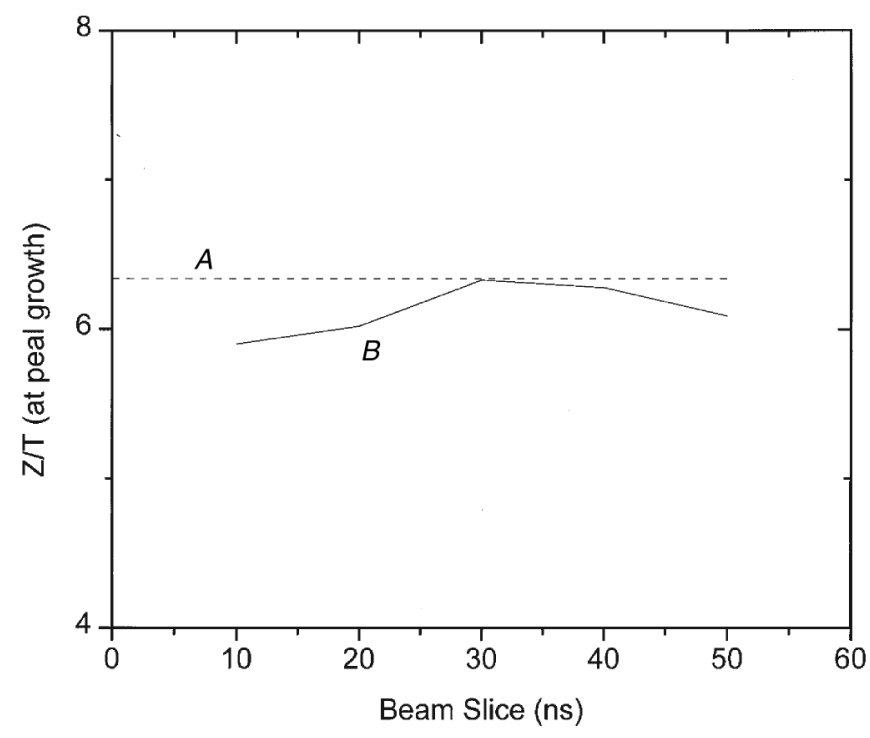

FIG. 2. For a Bennett conductivity profile, position of peak growth (or wave packet convection rate) from $(A)$ dispersion analysis and $(B)$ numerical integration of model equations. 


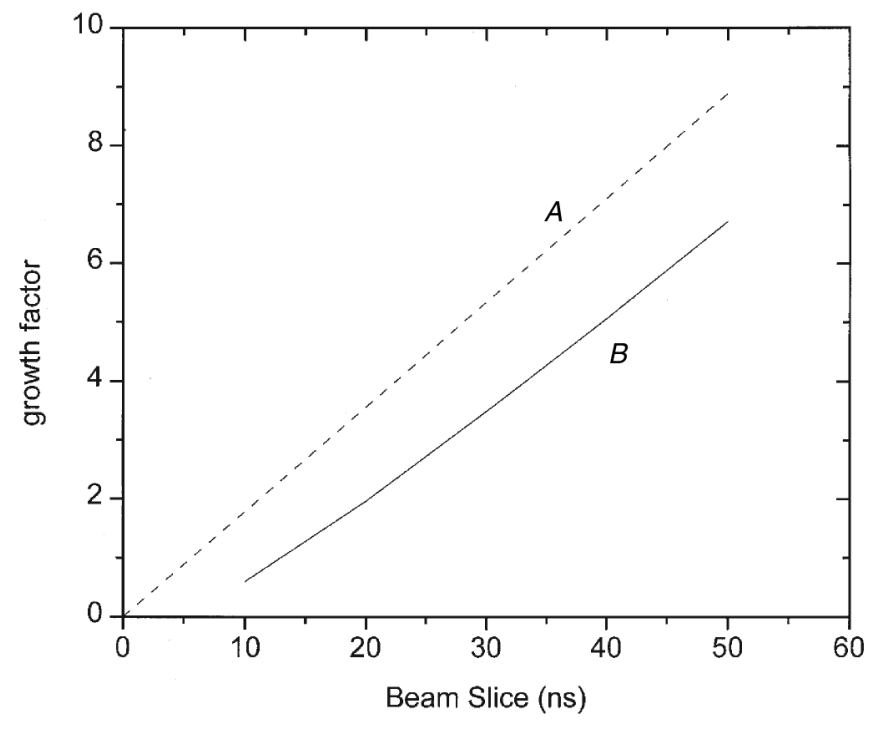

FIG. 3. Same as Fig. 1 for Lorentzian channel conductivity profile.

$$
\sigma(r, \tau)=\frac{n_{e}(r, \tau) e^{2}}{m_{e} \nu_{m}}
$$

In Eq. (28), $C_{1}$ depends on the beam energy and the propagation medium (1-atm air for our case) and determines the rate of primary ionization, while $C_{2}$ determines the temperature dependent rate of dissociative recombination. In Eq. (29), $\nu_{m}$ is the electron-neutral momentum transfer frequency which is both pressure and temperature dependent.

Using parameter values appropriate for a $300-\mathrm{MeV}, 2-$ $\mathrm{kA}, 2-\mathrm{cm}$ radius proton beam, Eq. (28) was solved numerically using Eq. (27) to specify the beam radius (and, hence, beam density) as a function of $\tau$. At the head of the beam,

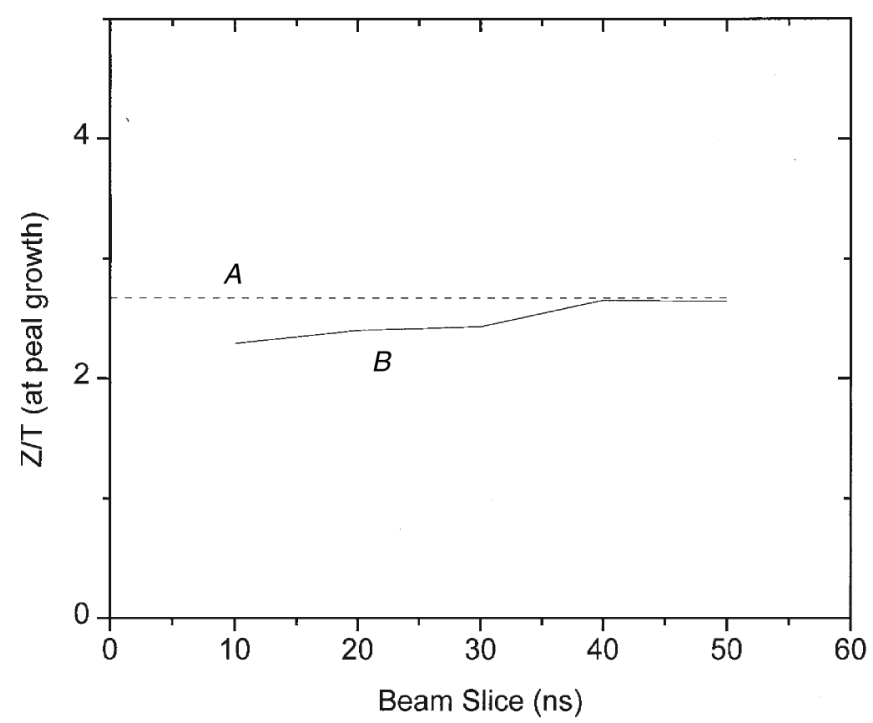

FIG. 4. Same as Fig. 2 for Lorentzian channel conductivity profile. the channel electron density was set to some nominally low value (a few times $10^{7}$ per $\mathrm{cm}^{3}$ ), and the channel was initialized to a Bennett profile. We see from Eq. (28) that for large $\tau$ (beam body) $n_{e}(r, \tau)$ evolves to a Lorentzian profile (square root of the Bennett). For the beam parameters above, we obtained an on-axis conductivity in the body of the beam of

$$
\frac{4 \pi \sigma_{0}}{c} \simeq 83.7 \mathrm{~cm}^{-1}
$$

a value consistent with those observed in more detailed numerical simulations. In summary, our solution evolves from a low conductivity Bennett profile at the head of the beam to a Lorentzian profile in the body [on-axis value given by Eq. (30)] with a characteristic evolution time of 4 ns.

Making use of the above solution for $\sigma(r, \tau)$, and using Eq. (27) to model the 3-to-1 radius tailoring, Eqs. (13) and (14) were solved numerically to determine instability growth for this time-dependent model of beam radius and channel conductivity. Here an oscillatory perturbation with a 2-ns period was applied at $z=0$, mimicking that used in particle simulations. (Only a weak dependence on the period of the perturbation was observed over the range 1 to $10 \mathrm{~ns}$.) Results are shown in Fig. 5 for the growth factor as a function of beam position $\tau$ for a constant $2-\mathrm{cm}$ beam radius and for 3-to- 1 radius tailoring. Curve $B$ of Fig. 5 represents larger growth factors than the constant radius, Lorentzian profile results of Fig. 3. This is due to the low conductivity at the beam head and the finite build-up time in $\tau$ according to Eqs. (27) and (28). The relatively low growth factors of curve $A$ (roughly $6 e$-foldings for the beam slice at $\tau=50 \mathrm{~ns}$ ) illustrate the dramatic reduction in instability growth achieved using radius tailoring at the

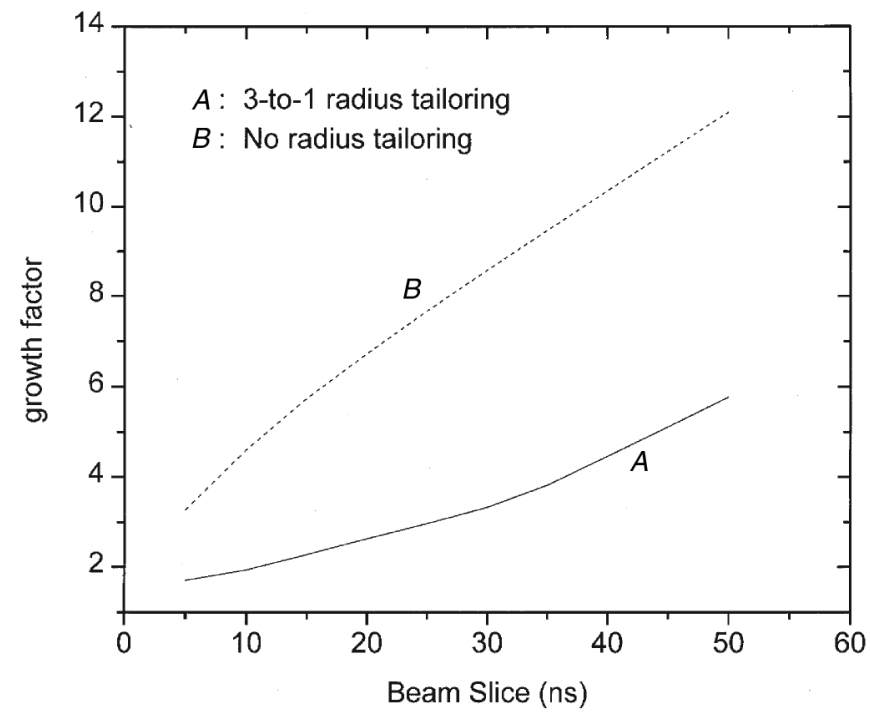

FIG. 5. Instability growth for a 300-MeV, 2-kA, 2-cm radius proton beam using the time-dependent channel conductivity model of Eqs. (27) and (28). 


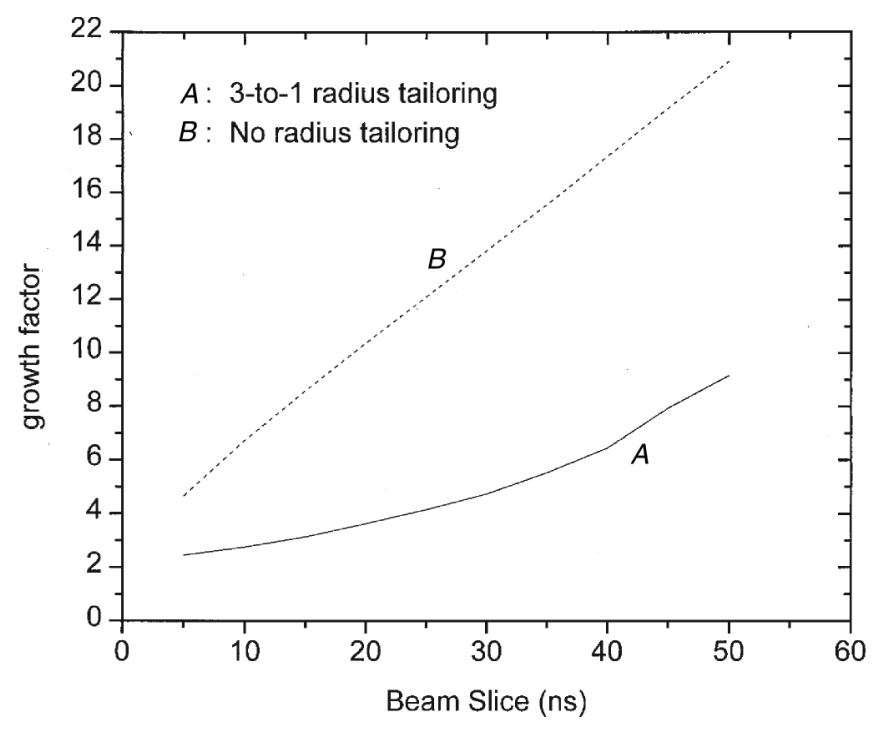

FIG. 6. Same as Fig. 5 for a $300 \mathrm{MeV}, 2 \mathrm{kA}, 1 \mathrm{~cm}$ radius proton beam.

beam head. For a 1-cm radius beam (see Fig. 6), and again for our beam slice at $\tau=50 \mathrm{~ns}$, slightly more than 9 $e$-foldings occur with 3-to-1 radius tailoring.

The numerical calculations above (carried out for a 300$\mathrm{MeV}$ beam) and comparison with the dispersion analysis demonstrate the consistency of the numerical model and suggest expected reductions in hose growth with more realistic conductivity profiles and with radius tailoring. Direct comparison with results of PIC simulations of proton beams at both $300 \mathrm{MeV}$ and $1 \mathrm{GeV}$ are presented in Secs. IVA and IV B below.

\section{SIMULATION MODEL}

A 3D PIC code is used with a hybrid model for tracking the evolution of the background gas conductivity and plasma currents. The simulations include models for charged particle energy loss and scattering in the ambient medium. The impact of these effects on proton beam propagation are addressed, along with beam head erosion, in Ref. [27].

The implicit three-dimensional simulations presented here are carried out in a moving frame of reference in $(r, z)$ coordinates with Fourier mode decomposition in the $\theta$ direction. Maxwell's equations in a frame moving at $v_{g}$ in the $z$ direction are (in dimensionless cgs units)

$$
\frac{\partial \vec{E}}{\partial t}=\frac{1}{\epsilon}\left(\vec{\nabla} \times \frac{\vec{B}}{\mu}-\vec{J}-\vec{J}_{p}\right)-v_{g} \frac{\partial \vec{E}}{\partial z},
$$

$$
\frac{\partial \vec{B}}{\partial t}=-\vec{\nabla} \times \vec{E}-v_{g} \frac{\partial \vec{B}}{\partial z}
$$

where $\vec{J}$ and $\vec{J}_{p}$ are the kinetic and fluid particle current densities. Regions can be specified with arbitrary electric permittivity $(\epsilon)$ and magnetic permeability $(\mu)$. The moving mesh is accounted for by the $v_{g} \partial / \partial z$ field convection terms.

For numerical stability, Eqs. (31) and (32) are integrated in time over time step $\Delta t$ with Eq. (31) multiplied by an integrating factor $\exp (\sigma t)$, where $\sigma$ is the local scalar conductivity. Variation in the third dimension is accounted for by Fourier analysis; $\partial / \partial \theta$ becomes $-m$ for cosines and $m$ for sines. The equation set in finite difference form is

$$
\begin{aligned}
E_{z}^{n+1}(S+\sigma \Delta t)= & E_{z}^{n} S+\left\{\frac{1}{r} \frac{\partial}{\partial r} r \frac{B_{\theta}}{\mu}-\frac{m}{r} \frac{B_{r}}{\mu}-J_{z}-Z n_{i} v_{z i}-\sigma\left[\frac{1}{n_{e}} \frac{\partial p_{e}}{\partial z}+\left(v_{z e}-v_{z i}\right) \nu_{m}+v_{r e} B_{\theta}-v_{\theta e} B_{r}\right]\right. \\
& \left.+\epsilon v_{g} \frac{\partial E_{z}}{\partial z}\right\}^{n+1 / 2} \frac{\Delta t}{\epsilon}, \\
E_{r}^{n+1}(S+\sigma \Delta t)= & E_{r}^{n} S+\left\{\frac{m}{r} \frac{1}{\mu} B_{z}-\frac{\partial\left(B_{\theta} / \mu\right)}{\partial z}-J_{r}-Z n_{i} v_{r i}-\sigma\left[\frac{1}{n_{e}} \frac{\partial p_{e}}{\partial r}+\left(v_{r e}-v_{r i}\right) \nu_{m}+v_{\theta e} B_{z}-v_{z e} B_{\theta}\right]\right. \\
& \left.+\epsilon v_{g} \frac{\partial E_{r}}{\partial z}\right\}^{n+1 / 2} \frac{\Delta t}{\epsilon}, \\
E_{\theta}^{n+1}(S+\sigma \Delta t)= & E_{\theta}^{n} S+\left\{\frac{\partial\left(B_{r} / m u\right)}{\partial z}-\frac{\partial\left(B_{z} / \mu\right)}{\partial r}-J_{\theta}-Z n_{i} v_{\theta i}-\sigma\left[-\frac{p_{e}}{n_{e}} \frac{m}{r}+\left(v_{\theta e}-v_{\theta i}\right) \nu_{m}+v_{z e} B_{r}-v_{r e} B_{z}\right]\right. \\
+ & \left.\epsilon v_{g} \frac{\partial E_{\theta}}{\partial z}\right\}^{n+1 / 2} \frac{\Delta t}{\epsilon},
\end{aligned}
$$




$$
\begin{aligned}
B_{z}^{n+1} & =-\left(\frac{1}{r} \frac{\partial}{\partial r} r E_{\theta}+\frac{m}{r} E_{r}+v_{g} \frac{\partial B_{z}}{\partial z}\right)^{n+1 / 2} \Delta t+B_{z}^{n}, \\
B_{r}^{n+1} & =\left(\frac{m}{r} E_{z}+\frac{\partial E_{\theta}}{\partial z}+v_{g} \frac{\partial B_{r}}{\partial z}\right)^{n+1 / 2} \Delta t+B_{r}^{n}, \\
B_{\theta}^{n+1} & =-\left(\frac{\partial E_{r}}{\partial z}-\frac{\partial E_{z}}{\partial r}+v_{g} \frac{\partial B_{\theta}}{\partial z}\right)^{n+1 / 2} \Delta t+B_{\theta}^{n} .
\end{aligned}
$$

The index $n$ represents the full time step and

$$
S=\frac{\sigma \Delta t}{\exp (\sigma \Delta t)-1} .
$$

Also, $p, n$, and $\nu$ are the pressure, density, and velocity of either the electron (subscript $e$ ) or the ion (subscript $i$ ) species and $Z$ is the ion charge state. The Ohm's law for plasma electron current includes electromagnetic, pressure gradient, and frictional forces. These equations are properly time centered with $n+1 / 2$ step determined by the average of the $n$ and $n+1$ values. Special care is taken for the $v_{g} \partial / \partial z$ term during the time integration. Because information cannot travel at speeds greater than $c$, the treatment for field $F$ at axial index $j$ is

$$
\begin{aligned}
\int v_{g} \frac{\partial F}{\partial z} d t= & v_{g} \frac{\Delta t}{\Delta z}\left[\left(1-v_{g}\right)\left(F_{j}-F_{j-1}\right)\right. \\
& \left.-\left(1+v_{g}\right)\left(F_{j+1}-F_{j}\right)\right] .
\end{aligned}
$$

Terms with conductivities (or $S$ ) account for mode couplings and convolutions.

Within the orthogonal coordinate system, Eqs. (33)(38) are solved iteratively using a dynamic form of the alternating direct implicit method [28]. Implicit solution in all coordinate directions can often result stable solution for time steps in excess of Courant condition. Particles are advanced implicitly in a manner similar to Ref. [29]. This technique can greatly reduce electron cyclotron and plasma frequency constraints as well as Debye length instability that is a well-known source of numerical heating.

The simulation geometry is 300 or $600-\mathrm{cm}$ long and the beam is continuously injected into the simulation volume until the beam head is within $50 \mathrm{~cm}$ of the downstream boundary. At this time, the computation mesh is moved forward at the beam speed. This is illustrated in Fig. 7. The main problem with PIC simulations of resistive hose is maintaining good statistics. Typical simulations use $\geq 10^{6}$ particles. However, significant noise remains, even with this large particle number. Thus, in the simulations, typical hose disturbances grow to a peak value, damp, and then some subsequent growth from noise is observed later.

The injected proton beam has a Bennett current-density profile of radius $a_{0}$, truncated at $2 a_{0}$. The beam current rises linearly from zero to peak current in $4 \mathrm{~ns}$. The injected protons are monoenergetic, with a transverse temperature $\left(\beta_{\perp}\right)$ that provides a near optimal matching condition. The a)

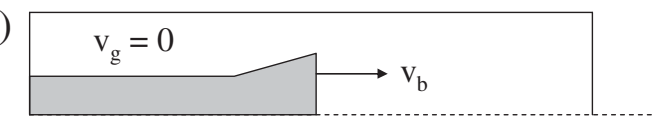

b)

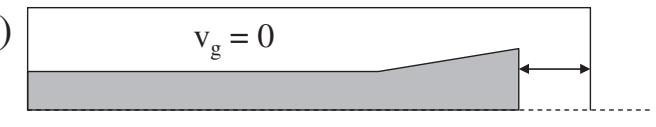

c)

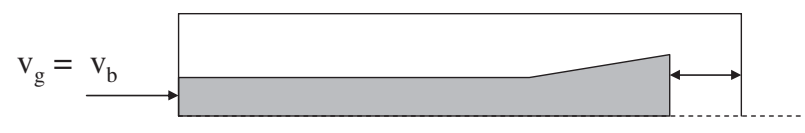

FIG. 7. Schematic illustrating the initialization of the simulations. (a) The beam with axial speed $v_{b}$ is injected into the stationary simulation box $\left(v_{g}=0\right)$. (b) The box remains stationary until the head beam is $50 \mathrm{~cm}$ from the end of the simulation region. (c) At this time the simulation box moves forward at the beam speed $\left(v_{g}=v_{b}\right)$.

injected beam is perturbed with a sinusoidal offset variation with an amplitude $A$ of $10^{-3} \mathrm{~cm}$ and a wavelength of $\lambda_{p}$. Values of $\lambda_{p}$ used in this report vary from 7.5 to $60 \mathrm{~cm}$ (roughly 300-2400 MHz frequency).

\section{SIMULATION RESULTS}

In this section, simulations are carried out and compared with the model presented in Sec. II. First, a series of simulations were carried out to examine the impact of different detuning ratios on the stability of the beam. These results are discussed in Sec. IVA. The resistive hose instability growth rate as a function of beam radius (at constant beam current) is discussed in Sec. IV B. Repeated beam pulses are known to reduce the density local to the beam. The stability of a single beam pulse in a reduced-density channel is discussed in Sec. IV C.

In our initial simulations the calculated dipole decay lengths were typically a factor 2-4 larger than calculated in Eq. (3) due to the evolution of non-Bennett conductivity profiles. This results in less growth with a faster convection of the wave packet. Thus, for a 30-ns-long beam, we need to propagate the beam $20-40 \mathrm{~m}$ to observe saturation for the entire pulse. So, typically the simulations presented here are set up to propagate the beam 50 to $100 \mathrm{~m}$.

\section{A. Detuning ratio}

The effect of "detuning" on the suppression of the resistive hose instability is well established for relativistic electron beams. Here, this technique is applied for a relativistic proton beam. Model calculations for four different detuning ratios are shown in the upper portion of Fig. 8 and the results of four simulations with the same detuning ratios are shown in the lower part of Fig. 8. For these calculations, the magnitude of the beam offset at a fixed distance back from the beam head $\xi$ (here $\xi \simeq 210 \mathrm{~cm}$ ) is shown for a 1-cm, 10-kA beam. The beam offsets from the four theory calculations are identical at early times. At this 


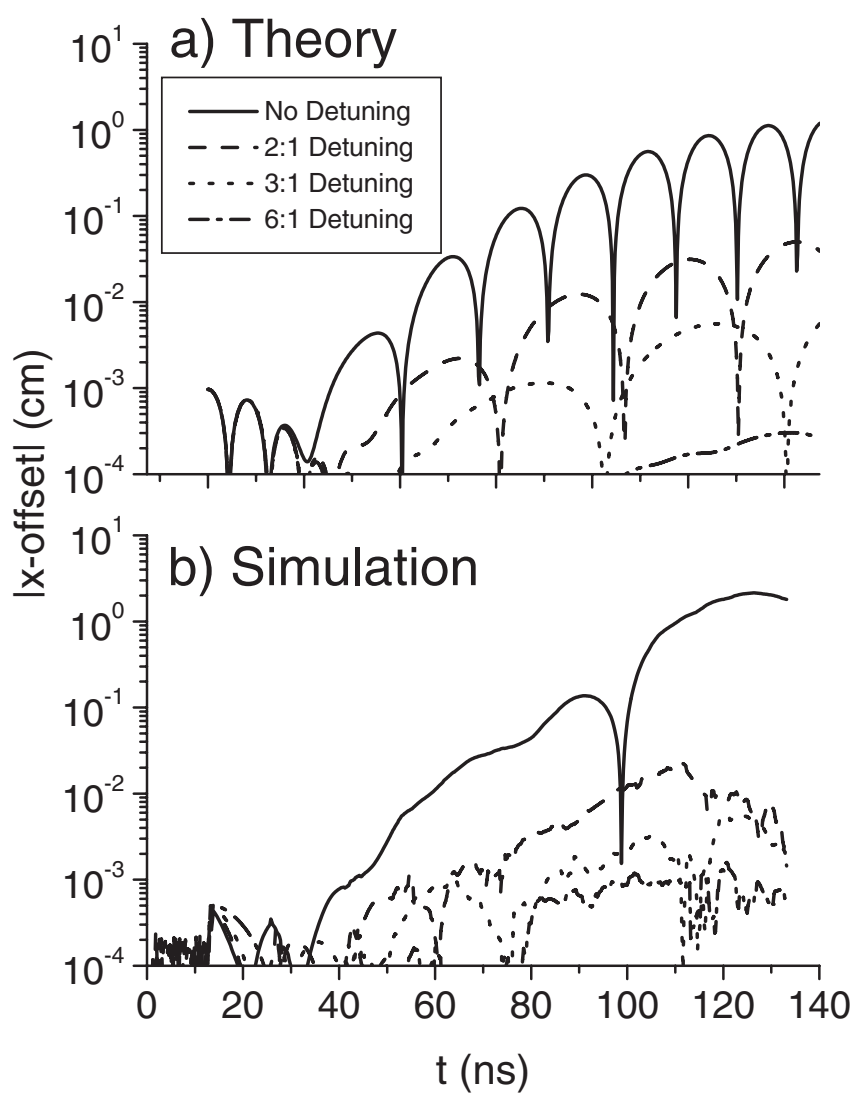

FIG. 8. Comparison of ion hose growth from (a) theory and (b) simulations with different detuning ratios. For these simulations $E_{b}=300 \mathrm{MeV}, a_{0}=1 \mathrm{~cm}, \lambda_{p}=15 \mathrm{~cm}, L=300 \mathrm{~cm}$, and $\xi=210 \mathrm{~cm}$.

distance back from the beam head, these calculated offsets characteristically decay from the initial offset for tens of nanoseconds before the resistive hose growth reaches this position back from the beam head. In the simulations, the initial offset is not immediately seen; the probe records the passage of the beam head first. When $210 \mathrm{~cm}$ of beam passes through this location (about $13 \mathrm{~ns}$ for these parameters), the probe then records the offset motion of a nearly constant slice of beam. Note that at this time, the beam offsets are falling, similar to the theory.

For larger detuning ratios, the beam offset is reduced dramatically, indicating a strong suppression of the resistive hose instability in both the model and the simulations.

The dipole decay times from the simulations in the body of the beams suggest that detuning ratios of 2:1 and 3:1 provide reasonable suppression of the resistive hose instability to short wavelength perturbations which grow in the beam head. Detuning ratios of 3:1 are used in the simulations presented in the remainder of this work.

\section{B. Beam radius}

Reducing the beam current-density can act to suppress the resistive hose growth by increasing $\tau_{B}$. A series of simulations with $E_{b}=1 \mathrm{GeV}$ were run and compared with the model. For all cases, $I_{b}=10 \mathrm{kA}$, and a 3:1 detuning ratio was used. Longer wavelengths tend to show larger growth of the resistive hose instability, so the simulation length $L$ was doubled from 300 to $600 \mathrm{~cm}$ for the simulations presented in the remainder of this section.

The theoretical predictions of the temporal evolution of the offset of a beam slice are shown for 1,2, and 4-cm radius beams in Fig. 9. The late-time growth rate scales as $1 / a_{0}$ as expected for the 2 and $4-\mathrm{cm}$ beams, but the $1-\mathrm{cm}$ beam has a late-time offset saturation that is similar to the $2-\mathrm{cm}$ result. Note that the oscillation periods are not constant in time and are different for each beam radius.

In Figs. 10-12 the theoretical predictions shown in Fig. 9 are compared individually with simulations at $\xi=$ $210-\mathrm{cm}$ slice location. The lower time axis for these plots is the simulation time, which includes data prior to the probe recording a constant beam slice, and the upper time axis is the theory time, where zero corresponds to the time at which the slice enters the model "box." The upper axis is shifted with respect to the lower in order to compare these two results.

The $a_{o}=1 \mathrm{~cm}$ simulations results show early-time offsets between 75 and $150 \mathrm{~ns}$ that are in good agreement with the theory. (Note that we use the lower time axis when referring to the graphs.) At later times the model shows saturation of the resistive hose growth, while in the simulation the offset continues to grow. This is due to numerical noise in the simulation model. This noise can be reduced (or delayed) to some extent by using more PIC macroparticles in the azimuthal direction. However, at present we are constrained by computational speeds and physical memory sizes.

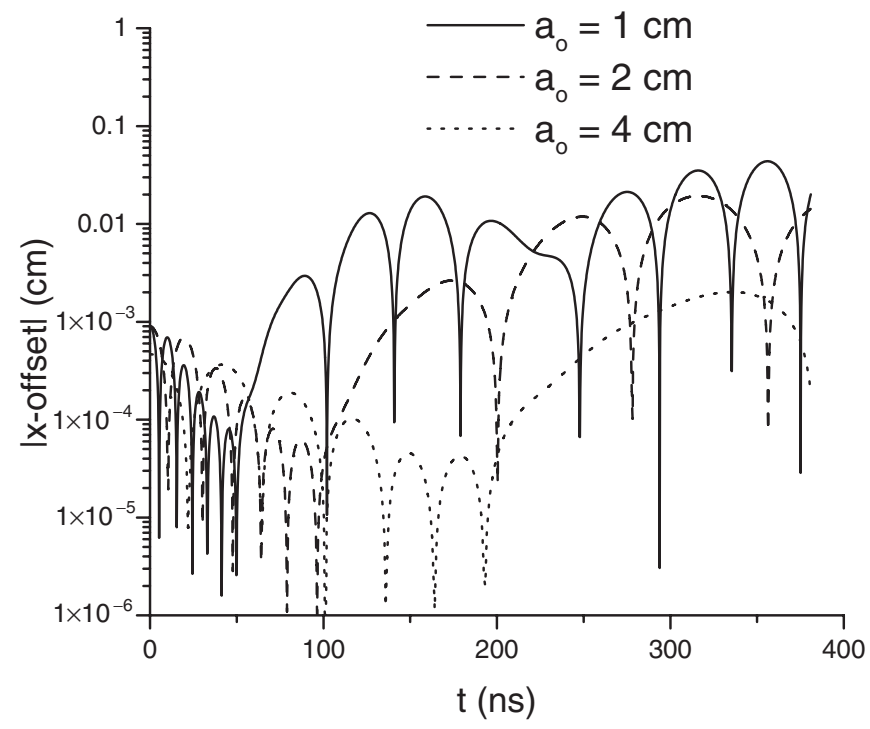

FIG. 9. Model predictions of hose growth with different beam radii for a beam slice that is $210 \mathrm{~cm}$ from the beam head. For these calculations $E_{b}=1 \mathrm{GeV}, \lambda_{p}=30 \mathrm{~cm}, A=0.001$, and a $3: 1$ detuning ratio is used. 


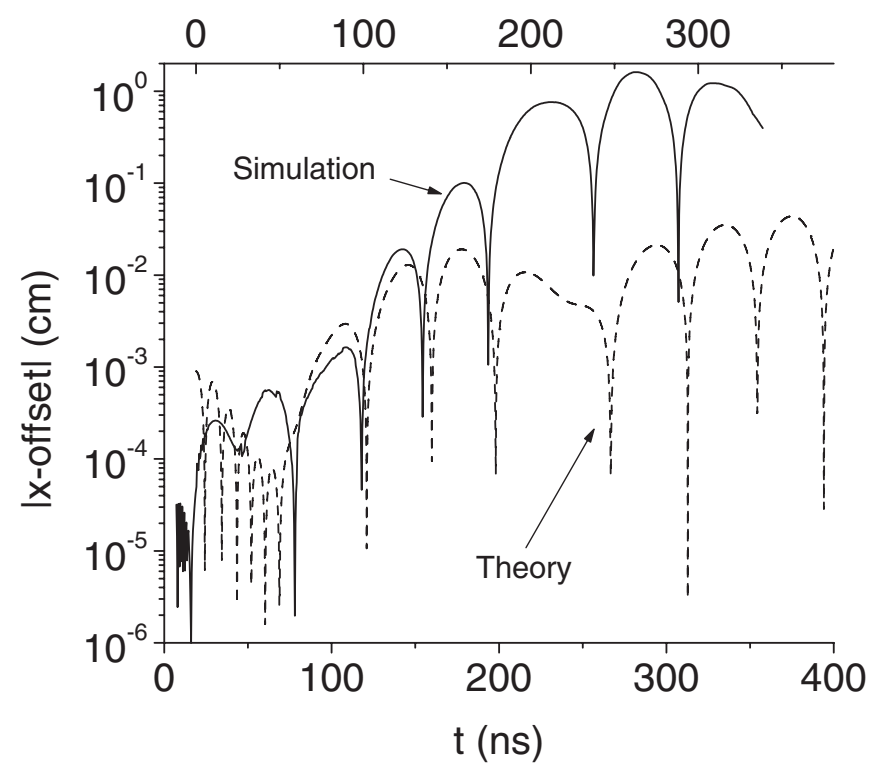

FIG. 10. Comparison of the model predictions (dashed line) with the results of a simulation (solid line) for $a_{0}=1 \mathrm{~cm}$.

The comparison for $a_{0}=2 \mathrm{~cm}$ shown in Fig. 11 is in good agreement for most of the calculation time. The overall trends are similar except for a large offset in the simulation around $100 \mathrm{~ns}$. The simulation results show somewhat more small amplitude noise than the $1-\mathrm{cm}$ results, due in part to numerical resolution of these small beam offsets.

Unlike the $a_{0}=1 \mathrm{~cm}$ results shown in Fig. 10, the period of the beam offset oscillations are not in good agreement. Again, this is due in part to the numerical resolution, which is a source of noise in the simulations.

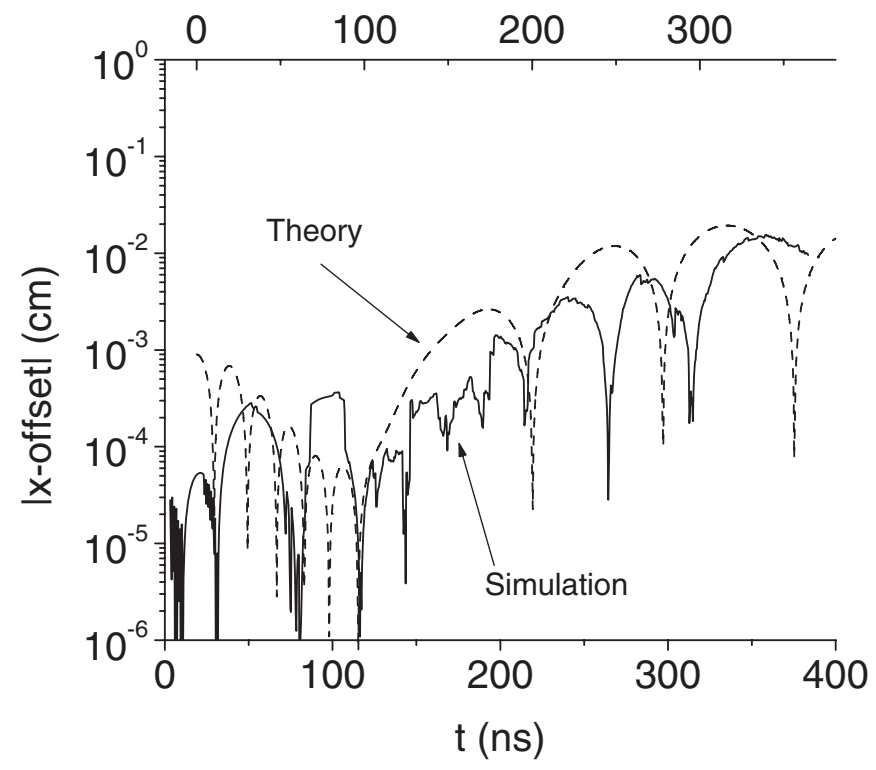

FIG. 11. Same as Fig. 10 except for $a_{0}=2 \mathrm{~cm}$.

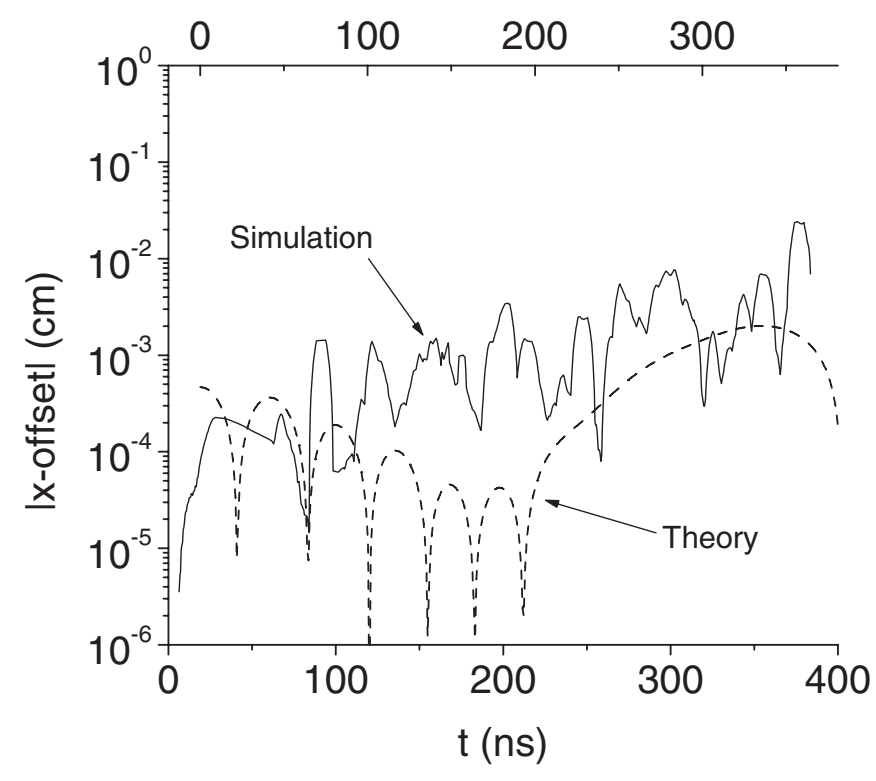

FIG. 12. Same as Fig. 10 except for $a_{0}=4 \mathrm{~cm}$.

Finally, the $a_{0}=4 \mathrm{~cm}$ results shown in Fig. 12 strongly reflect the numerical limitations of the simulation. Although the late-time amplitudes of the offsets are in reasonable agreement, the temporal evolution of the hose instability is not accurately tracked in the simulation. However, refinements in the simulations, especially near the axis may result in more accurate tracking of the beam offsets by reducing signal-to-noise ratios.

\section{Reduced-density channels}

A simulation was carried out with a reduced-density channel. The radial density profile used in the simulation

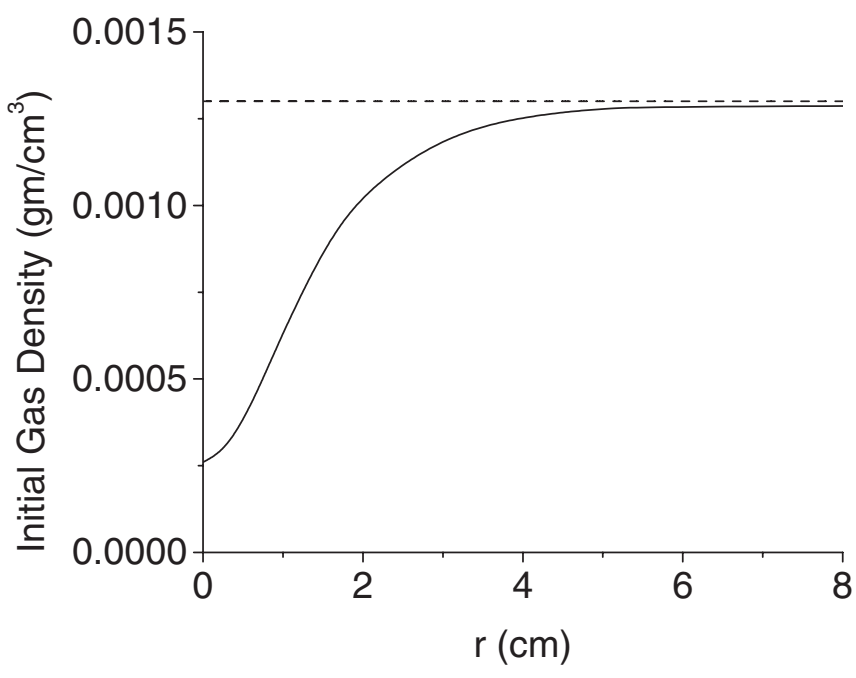

FIG. 13. Initial neutral gas density profile for reduced-density channel simulation (solid line) and nominal gas density profile (dashed line). 
is shown in Fig. 13. The beam parameters are $E_{b}=1 \mathrm{GeV}$, $a_{0}=2 \mathrm{~cm}$, and a 3-to- 1 detuning ratio is used.

Simulations with and without the reduced-density channel showed only small differences in the magnitude of the beam offset for $\xi=210 \mathrm{~cm}$. For these $1 \mathrm{GeV}$ beams, the current does not bunch up as was observed in the $300-\mathrm{MeV}$ channel simulations. Also, the peak $E / p$ values on axis in the $1-\mathrm{GeV}$ channel simulations are on the order of the electron avalanche threshold of $75 \mathrm{kV} / \mathrm{cm}$-atm. This suggests that the minimum channel depth that can be tolerated is about $0.1 \mathrm{~atm}$.

\section{SUMMARY}

We have developed a relatively simple and fast-running model capable of correctly identifying trends in hose growth behavior with variation in beam parameters (energy, radius, and radius tailoring). The model predictions have been compared with 3D PIC simulations and good agreement has been found over a range of beam parameters. These results indicate that these proton beams are particularly susceptible to long wavelength perturbations that grow in the beam body. For a $10-\mathrm{kA}, 2-\mathrm{cm}, 300-\mathrm{MeV}$ beam, the simulations results give an $e$-folding time of roughly $200 \mathrm{~cm}$ or $10 \mathrm{~ns}$. If $5 e$-foldings can be tolerated, one can expect to have beam radius growth for the nonlinear hose in $50 \mathrm{~ns}$. This sets a limit on the usable beam pulse length.

In a more extensive set of analytic and numerical calculations for relativistic electron beams, Lampe et al. [6] showed that the two effects tend to cancel one another. This may in part explain the success of our rudimentary model in reproducing the trends observed in the detailed PIC simulations presented in Sec. IV.

\section{ACKNOWLEDGMENTS}

The authors acknowledge helpful technical discussions with Dr. R. Turman, Sandia National Laboratories. Portions of the research presented here were carried out while several of the authors (D. V.R., T.C.G., D. R. W.) were with Mission Research Corporation in Albuquerque, New Mexico. This work was supported by Sandia National Laboratories. Sandia is a multiprogram laboratory operated by Sandia Corporation, a Lockheed-Martin Company, for the United States Department of Energy, under Contract No. DE-AC04-94AL85000.

[1] E. P. Lee, Phys. Fluids 21, 1327 (1978).

[2] K. G. Moses, R. W. Bauer, and S. D. Winter, Phys. Fluids 16, 436 (1973).
[3] E. J. Lauer, R. J. Briggs, T. J. Fessenden, R. E. Hester, and E. P. Lee, Phys. Fluids 21, 1344 (1978).

[4] H. S. Uhm and M. Lampe, Phys. Fluids 23, 1574 (1980).

[5] H. S. Uhm and M. Lampe, Phys. Fluids 25, 1444 (1982).

[6] M. Lampe, W. Sharp, R. F. Hubbard, E. P. Lee, and R. J. Briggs, Phys. Fluids 27, 2921 (1984).

[7] T. W. L. Sanford, J.A. Halbleib, W. H. McAtee, R. C. Mock, J. W. Poukey, and D. R. Welch, J. Appl. Phys. 70, 1778 (1991).

[8] J. G. Siambis, Phys. Fluids B 4, 3390 (1992).

[9] T. W. L. Sanford, D. R. Welch, and R.C. Mock, Phys. Fluids B 5, 4144 (1993).

[10] T. W. L. Sanford, D. R. Welch, and R.C. Mock, Phys. Plasmas 1, 404 (1994).

[11] M. C. Myers, R. F. Fernsler, R. A. Meger, J. A. Antoniades, D. P. Murphy, and R. F. Hubbard, J. Appl. Phys. 80, 4258 (1996).

[12] R. F. Fernsler, S. P. Slinker, M. Lampe, and R. F. Hubbard, Phys. Plasmas 2, 4338 (1995).

[13] H.S. Uhm and R. C. Davidson, IEEE Trans. Plasma Sci. 33, 1395 (2005).

[14] D. R. Welch, F. M. Bieniosek, and B. B. Godfrey, Phys. Rev. Lett. 65, 3128 (1990).

[15] D. P. Murphy, M. Raleigh, R. E. Pechacek, and J. R. Greig, Phys. Fluids 30, 232 (1987).

[16] D. P. Murphy, R. E. Pechacek, D. P. Taggert, R. F. Fernsler, R. F. Hubbard, S. P. Slinker, and R. A. Meger, Phys. Fluids B 4, 3407 (1992).

[17] M. C. Myers, J. A. Antoniades, R. A. Meger, D. P. Murphy, R. F. Fernsler, and R. F. Hubbard, J. Appl. Phys. 78, 3580 (1995).

[18] R.F. Fernsler, S. P. Slinker, and R.F. Hubbard, Phys. Fluids B 3, 2696 (1991).

[19] J.-M. Dolique and M. Khodja, Laser Part. Beams 11, 685 (1993).

[20] E. K. Kolesnikov and A. S. Manuilov, Tech. Phys. 42, 648 (1997).

[21] D. R. Welch, C.L. Olson, and T.W.L. Sanford, Phys. Plasmas 1, 764 (1994).

[22] P. F. Ottinger, D. V. Rose, and C. L. Olson, J. Appl. Phys. 75, 4402 (1994).

[23] D. R. Welch, M.E. Cuneo, C.L. Olson, and T. A. Mehlhorn, Phys. Plasmas 3, 2113 (1996).

[24] K. Hahn and E. Lee, Fusion Eng. Des. 32-33, 417 (1996).

[25] D. R. Welch, T. C. Genoni, D. V. Rose, B. V. Oliver, R. E. Clark, C. L. Olson, and S. S. Yu, Phys. Plasmas 10, 2442 (2003).

[26] F. W. Chambers and D. M. Cox, Lawrence Livermore National Laboratory Report No. UCID-19213.

[27] D. V. Rose, T. C. Genoni, and D. R. Welch, Phys. Plasmas 9, 1053 (2002).

[28] D. W. Hewett, D. J. Larson, and S. Doss, J. Comput. Phys. 101, 11 (1992).

[29] D. W. Hewett and A. B. Langdon, J. Comput. Phys. 72, 121 (1987). 